\section{Trajectory of brain maturation and sex-specific cognitive abnormalities in early-onset psychosis}

Rev. Bras. Psiquiatr. 2017;39:381

doi:10.1590/1516-4446-2017-3903

Ruiz-Veguilla et al., ${ }^{1}$ in the January-March 2017 issue of Revista Brasileira de Psiquiatria, reported on "sexspecific cognitive abnormalities in early-onset psychosis". The study makes a contribution to our understanding of how schizophrenia might influence brain maturation and function. However, the authors based their conclusion on indirect evidence. The authors posited that the earlier peak of prefrontal cortex maturation seen in non-schizophrenia adolescent females will not be seen in female children and adolescents with first episode early onset psychosis (EOP). They also hypothesized that absence of the earlier peak of prefrontal cortex maturation in female children with EOP would result in no sex differences in working memory in the EOP population. Even though the authors confirmed their hypothesis in their sample, a number of important issues are worthy of discussion.

First, what is the exact nature of the sex-specific abnormalities in cognitive function that the authors concluded were present in their sample of patients with EOP? There were no sex differences in verbal working memory and auditory attention tests in the EOP sample and it appears that they based their conclusion on the finding that group effect (i.e. control vs. EOP) on verbal working memory and auditory attention was modified by sex. However, this interaction might just mean that sex has an effect on cognition in controls but not patients. Furthermore, at the end of the first paragraph in the discussion section, the authors wrote that "less impairment in verbal working memory and auditory attention was present only in girls with EOP" but this statement is not supported by Table 4 of the paper. An important question that should also be asked is: does schizophrenia slow brain maturation at the same rate in female and male adolescent patients? As acknowledged by the authors, their study cannot answer this question due to its cross-sectional design.

In terms of future direction, it would be desirable for Ruiz-Veguilla et al. to follow this cohort of patients and controls longitudinally (and if possible increase the sample size). Longitudinal follow-up with serial assessments of cognitive function as well as imaging modalities will be helpful to elucidate the nature of any sex-specific cognitive abnormality in early-onset psychosis and underlying mechanisms by which schizophrenia affects brain maturation. It may also be beneficial to assess cognition with the MATRICS Consensus Cognitive Battery, which was designed to ensure that cognitive testing is consistent and comparable across studies. ${ }^{2}$
Olaoluwa O. Okusaga

Harris County Psychiatric Center, Department of Psychiatry and Behavioral Sciences, McGovern Medical School, University of Texas Health Science Center at Houston, Houston, TX, USA

Submitted Apr 20 2017, accepted Apr 232017.

\section{Disclosure}

The author reports no conflicts of interest.

\section{References}

1 Ruiz-Veguilla M, Moreno-Granados J, Salcedo-Marin MD, Barrigon $\mathrm{ML}$, Blanco-Morales MJ, Igunza E, et al. Sex-specific cognitive abnormalities in early-onset psychosis. Rev Bras Psiquiatr. 2017;39: 28-35.

2 Marder SR, Fenton W. Measurement and treatment research to improve cognition in schizophrenia: NIMH MATRICS initiative to support the development of agents for improving cognition in schizophrenia. Schizophr Res. 2004;72:5-9.

\section{Zika virus infection followed by a first episode of psychosis: another flavivirus leading to pure psychiatric symptomatology}

Rev. Bras. Psiquiatr. 2017;39:381-382

doi:10.1590/1516-4446-2017-2308

Zika virus (ZIKV), a flavivirus primarily transmitted by Aedes mosquitoes, represents a major public health concern. ZIKV infection, previously considered a self-limited febrile exanthematic disease, leads to serious neurologic complications. ${ }^{1}$

Microcephaly and extensive brain damage can result from congenital ZIKV infection. An association with GuillainBarre Syndrome was suggested after the French Polynesia outbreak, ${ }^{2}$ and reports from endemic areas suggest that acute ZIKV infection leads to numerous central nervous system (CNS) complications. Considering the complexity of CNS function, we can expect a variety of clinical manifestations, even purely psychiatric symptoms.

A 17-year-old boy was transferred to our psychiatric emergency ward for evaluation of a first-episode psychosis (FEP). He had no significant health history or previous psychiatric history. Ten days prior to admission, he suddenly presented paranoid delusions and vivid auditory, somatic and olfactory hallucinations. He showed intense anxiety and panic-like symptoms, alternating with moments of inadequate behavioral disinhibition. Symptoms also included sleeplessness, increased speech production, vocal mannerisms and refusal to eat. A distinct period of altered mood was negated. Upon admission he was fully conscious with no attention deficits, disoriented 